\title{
Comparison of Area 17 Cellular Composition in Laboratory and Wild-Caught Rats Including Diurnal and Nocturnal Species
}

\author{
Katharine L. Campi ${ }^{a, b}$ Christine E. Collins ${ }^{c}$ William D. Todd ${ }^{d} \quad$ Jon Kaas ${ }^{c}$ \\ Leah Krubitzer ${ }^{\mathrm{a}, \mathrm{b}}$ \\ ${ }^{a}$ Center for Neuroscience and ${ }^{b}$ Department of Psychology, University of California, Davis, Calif., \\ 'Department of Psychology, Vanderbilt University, Nashville, Tenn., and d Department of Psychology, \\ University of lowa, lowa City, lowa, USA
}

\section{Key Words}

Architecture $\cdot$ Area $17 \cdot$ Cellular composition • Evolution • Isotropic fractionator $\cdot$ Vision

\begin{abstract}
In this study we examine the size of primary sensory areas in the neocortex and the cellular composition of area 17/V1 in three rodent groups: laboratory nocturnal Norway rats (Long-Evans; Rattus norvegicus), wild-caught nocturnal Norway rats (R. norvegicus), and laboratory diurnal Nile grass rats (Arvicanthis niloticus). Specifically, we used areal measures of myeloarchitecture of the primary sensory areas to compare area size and the isotropic fractionator method to estimate the number of neurons and nonneurons in area 17 in each species. Our results demonstrate that the percentage of cortex devoted to area 17 is significantly greater and the percentage of cortex devoted to $\mathrm{S} 1$ is significantly smaller in the diurnal Nile grass rat compared with the nocturnal Norway rat groups. Further, the laboratory rodent groups have a greater percentage of cortex devoted to auditory cortex compared with the wild-caught group. We also demonstrate that wild-caught rats have a greater density of neurons in area 17 compared to laboratory-reared animals. However, there were no other clear cellular composition differences in area 17 or differences in the percentage of brain weight de-
\end{abstract}

voted to area 17 between nocturnal and diurnal rats. Thus, there are differences in primary sensory area size between diurnal versus nocturnal and laboratory versus wild-caught rat groups and cellular density between wild-caught and laboratory rat groups. Our results demonstrate that the differences in the size and cellular composition of cortical areas do not fit with what would be expected based on brain scaling differences alone, and have a consistent relationship with lifestyle and sensory morphology.

Copyright $\odot 2011$ S. Karger AG, Base

\section{Introduction}

Recent anatomical data in our laboratory indicate that the percentage of cortex devoted to a specific sensory modality varies with both diel pattern (day/night activity cycle) and rearing condition in rodents [Campi and Krubitzer, 2010]. Not surprisingly, diurnal squirrels have a larger percentage of cortex devoted to visual areas compared to nocturnal rats, which have a larger percentage of cortex devoted to somatosensory and auditory areas. Although laboratory rats have a larger percentage of cortex devoted to somatosensory and auditory areas compared to the wild-caught rats, there is no difference in the percentage of cortex devoted to the visual areas or to sensory/

\section{KARGER \\ Fax +4161306 1234 \\ E-Mail karger@karger.ch}

www.karger.com (c) 2011 S. Karger AG, Basel

Accessible online at: www.karger.com/bbe
Leah Krubitzer

Center for Neuroscience

1544 Newton Ct

Davis, CA 95616 (USA)

Tel. +1 530757 8868, E-Mail lakrubitzer@ucdavis.edu 
motor cortex versus nondelineated cortex between the two rat groups. This result was surprising in light of evidence from environmental enrichment studies showing increases in synapses and brain volume in rodent brains that had experienced enriched environments, and from volumetric brain studies which indicate that wild animals have larger brains compared to their domestic counterparts [Ebinger, 1974; Kruska, 1988; Mohammed et al., 2002; Faherty et al., 2003; Kolb et al., 2003; Gelfo et al., 2009]. However, the wild versus domestic comparisons in these studies were of volume differences for either the entire brain or gross structures (cortex, cerebellum and remaining areas), whereas our study compared smaller subdivisions of the brain, such as the size of individual cortical sensory areas. These differences in results of studies of gross brain size and cortical field size results are interesting because they indicate that evolutionary alterations to brains across hierarchical levels are nonlinear. In other words, changes in field size do not necessarily translate into commensurate changes in overall brain size, indicating a mosaic or patchwork pattern of brain evolution specific to the combination of environmental, behavioral and morphological demands operating with some degree of independence on each level of organization.

In the present investigation, we extend our comparisons of visual cortex in laboratory and wild-caught rodents to a cellular level of organization and use the isotropic fractionator method [Herculano-Houzel and Lent, 2005 ] to examine the number and density of neurons and nonneurons in the primary visual cortex (V1/area 17) in the laboratory Norway rat (Rattus norvegicus, nocturnal), wild-caught Norway rat ( $R$. norvegicus, nocturnal) and laboratory Nile grass rat (Arvicanthis niloticus). These species afford two interesting comparisons. First, they allow us to compare relatively closely related nocturnal and diurnal species: the Norway rat and Nile grass rat, respectively (fig. 1). Most comparisons of nocturnal and diurnal rodent cortical organization have been made between squirrels and mice or rats. While these comparisons are important, Muridae and Sciuridae rodents have been evolving independently for about 70 million years [Steppan et al., 2004; Roll et al., 2006; Huchon et al., 2007]. On the other hand, Norway rats and Nile grass rats are separated by approximately 10 million years [Chevret et al., 1993]. Thus, these comparisons may better reveal alterations to the nervous system due to the acquisition of diurnal vision.

The second reason these comparisons are important is because they allow us to compare the cellular organiza- tion in the same species ( $R$. norvegicus, with some strain differences) in laboratory versus wild-caught rats. The laboratory rat was domesticated through mixtures between pet store rats and wild-caught Norway rats during the 1880s. Since then, several stocks or strains of laboratory rats have been inbred or outbred for specific characteristics, such as obesity or hypertension in the Zucker strain or the multipurpose albino Sprague-Dawley strain. We chose the Long-Evans rat because it is an outbred strain with normal (black 'hood' on white body) pigmentation and presumably with a visual system more similar to that of the wild-caught Norway rat than the albino strains. This strain was developed through the mixture of Wistar strain females with wild-caught Norway rats in 1915 by Drs. Long and Evans.

Much is known about the visual system of laboratory rats at all levels of processing. Although the visual system has not been as extensively examined in the Nile grass rat, differences in retinal composition and visual acuity between the two groups have been established. The Nile grass rat has a retina composed of $30-40 \%$ cones and has a visual acuity of 1.3 cycles per degree [Gaillard et al., $2008,2009]$. In contrast, the laboratory Norway rat has a retina composed of $1-3 \%$ cones and has a visual acuity of $\leq 0.54$ cycles per degree [Douglas et al., 2005]. The Nile grass rat has the same type of cone photoreceptors as other murine rodents; the M/L cones are maximally responsive to $510 \mathrm{~nm}$ wavelength and shortwave length cones are responsive in the UV range [Gilmour et al., 2008]. It is possible that the acuity difference between these nocturnal and diurnal rat groups is due mainly to these retinal differences, but there have been no comparisons at higher levels of organization in these different groups of rats.

Here, we compare the primary visual area, V1 or area 17 , in these three species of rats in order to determine the extent to which cellular composition is altered by diel pattern and rearing condition. Specifically, we examine differences in overall cortical area size, as well as neuronal and nonneuronal cell number and density of cells in area 17. These comparisons will allow us to appreciate the relationship between overall brain size, cortical field size and cellular composition within a cortical field, and how different levels of organization coevolve to generate differences in sensory-driven behavior. Further this will allow a comparison between rats reared in a standard laboratory environment with rats reared in enriched environments. Previous studies examining neuroplasticity due to environmental enrichment have demonstrated changes at all levels of organization from molecular to behavioral, 
Fig. 1. Phylogenetic tree of the order Rodentia with representative individuals of each family listed. The names of the rodent groups examined in this study are set off here by larger font. Numbers reference time in millions of years ago (mya) to the present for the split of each group are listed. Taken from Huchon et al. [2002, 2007] and Steppan et al. [2004].

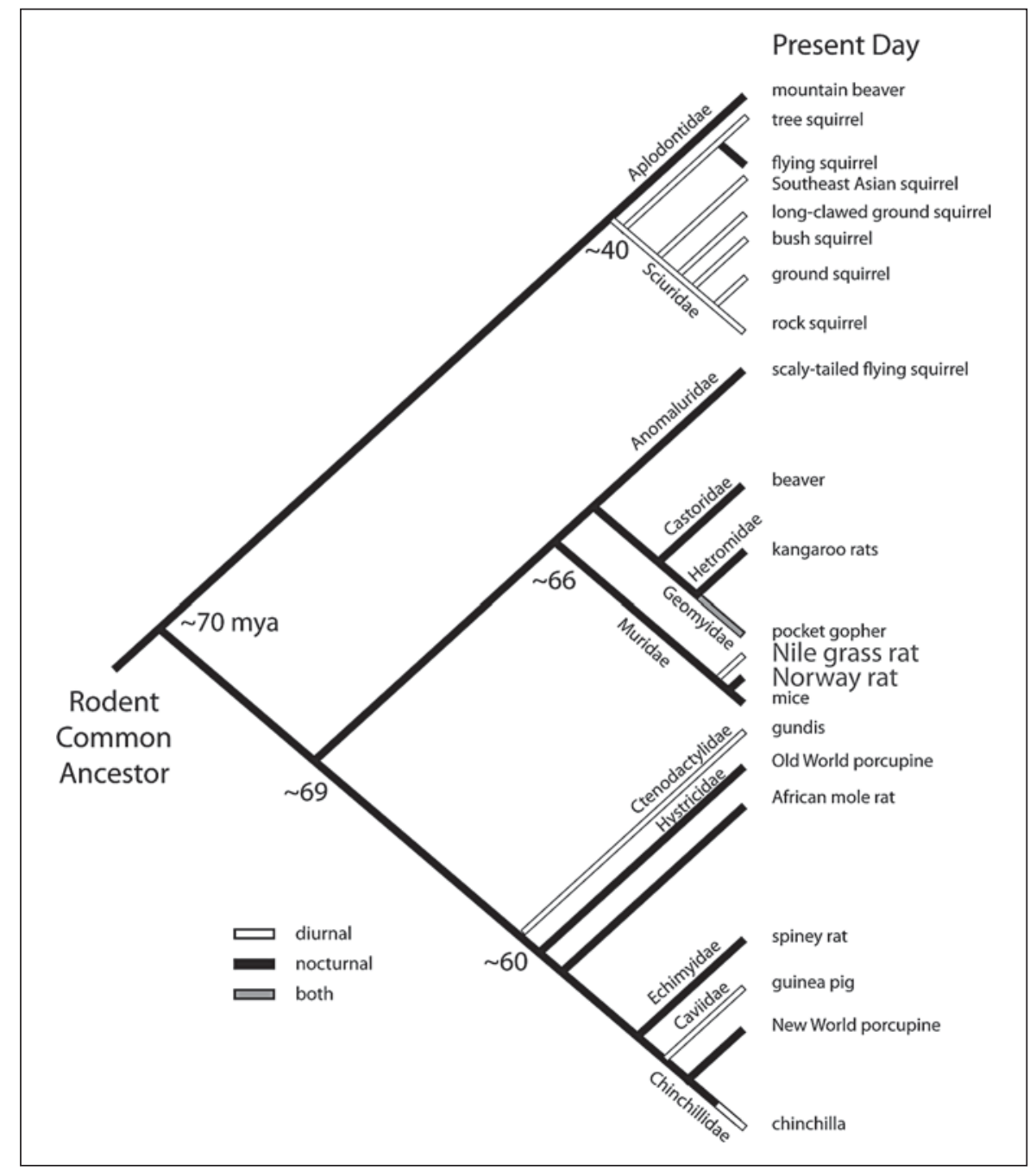

including increases in brain-derived neurotrophic factor and acetylcholinesterase [Rosenzweig, 1966; Cancedda et al., 2004], smaller, nonoverlapping receptive fields and an increase in the areal extent of the enriched receptor array [Coq and Xerri, 1998]. In the visual cortex, cats reared in enriched environments have a higher proportion of orientation-selective cells [Beaulieu and Cynader, 1991] and environmentally enriched mice a higher visual acuity [Prusky et al., 2000] than mice and cats reared in impoverished environments. Thus, in addition to genetic factors that contribute to the establishment, composition and plasticity of cortical areas, the sensory environment in which an animal is reared plays a large role on the resulting cortical organization and the behavior it generates [for cortical patterning review, see Krubitzer and Kahn, 2003; for enrichment reviews, see Arai and Feig,
2010; Baroncelli et al., 2010, and Diamond, 2001]. The current study takes a novel approach to addressing these issues by directly comparing the cellular composition in two groups of species that have been reared in dramatically different environments (natural versus laboratory), and in two closely related species that have undergone genetic modifications to the visual system associated with nocturnality and diurnality, that have been reared in a similar laboratory setting.

\section{Materials and Methods}

Twelve cortical hemispheres from 6 (3 female) Norway rats (laboratory, R. norvegicus), 10 hemispheres from 5 ( 4 female) Norway rats (wild-caught, $R$. norvegicus), and 13 hemispheres from 7 (2 female) Nile grass rats (A. niloticus) were used for histological 
Table 1. Weight comparisons for body, brain, area 17, and area 17 percentage of brain weight

\begin{tabular}{lllll}
\hline & Body mass, $g$ & Brain mass, $g$ & Area 17, g & Area 17, \% wt \\
\hline Laboratory Norway rat & $508.3 \pm 150.2$ & $1.827 \pm 0.049$ & $0.022 \pm 0.002$ & $0.04 \pm 0.013$ \\
Wild-caught Norway rat & $170.1 \pm 16.6$ & $1.541 \pm 0.053$ & $0.018 \pm 0.001$ & $0.04 \pm 0.009$ \\
Nile grass rat & $104.3 \pm 9.3$ & $0.872 \pm 0.024$ & $0.010 \pm 0.0004$ & $0.04 \pm 0.005$ \\
\hline Difference & 5-fold & 2-fold & 2-fold & no difference \\
\hline
\end{tabular}

comparisons. The Nile grass rat brains were shipped to our lab from another lab and one hemisphere arrived with too much damage for use in the study. For a complete listing of means for body, brain and area 17 weights for each group, see table 1 . Wildcaught rats were visually inspected to ensure that there were no injuries or deformities, particularly of the eyes, ears, paws and whiskers. Regarding the correct classification of our wild-caught rat group, several identifying features were examined in order to categorize our specimens first as $R$. norvegicus and then as adults: body/tail length ratio, body weight, fur color and texture, dentition, bands per inch of tail as well as ecological factors observed during trapping. Although we could not determine the age of the wild-caught animals, their weights and size indicated that they were adults. Specifically, rats are sexually mature by $3-4$ months of age and although we cannot know the age of our wild rats, studies have been done to correlate sexual maturity with weight. In a study on the sexual maturity of the wild rat $[R$. norvegicus; Perry, $1946]$, the presence and number of corpora lutea were used to identify the weight at which female rats are sexually mature and it was observed that $50 \%$ of rats had corpora lutea at $150 \mathrm{~g}$. Another study examining the differences between city and farm rats found that $50 \%$ of rats were sexually mature using vaginal perforation as the measure at $88 \mathrm{~g}$ for farm rats and $105 \mathrm{~g}$ for city rats [Davis, 1947]. As 4 of 5 of our wild-caught rats were females with an average weight of $170 \mathrm{~g}$, we concluded that they were adults. Norway rats (laboratory) ranged from 9 to 12 months in age and Nile grass rats ranged from 11 to 17 months in age. All procedures were approved by the Internal Animal Care and Use Committee (IACUC) and conformed to NIH guidelines.

\section{Histological Processing for Cell Composition}

Animals were euthanized with a lethal dose of sodium pentobarbital $(250 \mathrm{mg} / \mathrm{kg})$ and perfused transcardially with $0.9 \%$ saline, followed by $3 \%$ paraformaldehyde ( $2 \%$ paraformaldehyde was used for the Nile grass rats) in $0.1 \mathrm{M}$ phosphate buffer ( $\mathrm{pH}$ 7.4). After fixation, the brain was extracted from the skull, the hemispheres were separated from the subcortical structures, the medial wall was reflected and then the entire hemisphere was flattened between two glass slides for 24-48 h. Once sufficiently flattened, the hemisphere was placed on a light box, and area 17 was visualized and dissected from the cortical sheet (fig. $2 \mathrm{a}-\mathrm{c}$ ). The dissected piece of cortex was photographed and weighed.

Dissected area 17 pieces were stored in $0.1 \mathrm{M}$ phosphate buffer until the time of processing. The isotropic fractionator method has been previously described in detail in Herculano-Houzel and Lent [2005]. Briefly, the dissected area 17 piece was placed in a Petri dish and finely chopped using a scalpel and then transferred to a glass Tenbroeck tissue grinder or potter. The tissue sample was then ground up with a small amount of dissociation fluid (sodium citrate, Triton X-100 and distilled water) until a suspension with no visible clumps was formed. The homogenate containing cellular nuclei was then transferred into $15-\mathrm{ml}$ Falcon tubes for further processing. Processing for the visualization of total nuclei was performed using 4',6-diamidino-2-phenylindole (DAPI; Invitrogen), a DNA stain that labels all cellular nuclei regardless of cell type, and anti-NeuN antibody (MAB 377; Millipore) that specifically labels neuronal nuclei that express neuronal nuclear antigen.

Although the isotropic fractionator method is relatively new, the individual techniques and equipment are not and compare well with older methods. Production of suspensions from fixed or fresh tissue samples using glass homogenizers identical to those used in the isotropic fractionator method are commonplace in many areas of science, including neuroscience, since the 1960s, and have generally been used by those investigating more molecular-oriented questions [e.g. Lovtrup-Rein and McEwen, 1966]. There is no evidence that nuclei are lost in the grinding process. Estimates of neuron numbers in the cortex of macaque monkeys using the isotropic fractionator method [e.g. Collins et al., 2010] are not significantly different from those estimated using the stereological optical fractionator method [Christensen et al., 2007]. Furthermore, based on rigorous testing, the World Health Organization specifically recommends the Neubauer chamber [WHO, 1999], the type of hemocytometer used in our study, for analysis of semen samples in fertility diagnoses. It has been tested against other counting chambers, and has been found to produce the most accurate and repeatable counts of the available hemocytometers [e.g. Sukcharoen et al., 1994]. When tested against other hemocytometers using the classic limits of agreement analysis method published by Bland and Altman [1986], the Neubauer chamber is rated as highly accurate in comparison to other chambers designed for the same purpose [e.g. Mahmoud et al., 1997]. All of these tests validating the counting methods for cells suspended in fluids predate the publication of the isotropic fractionator paper [Herculano-Houzel and Lent, 2005].

\section{Counting of Nuclei and Data Analysis}

The suspensions of nuclei were vortexed and $10-\mu l$ aliquots were loaded into a Neubauer cell-counting chamber (Optik Labor) and placed on a fluorescence microscope for visualization and counting of nuclei (fig. 2d-f). Standard stereological protocols were used [Mouton, 2002]. Estimates of total nuclei were derived from nucleus counts from 16 squares of the Neubauer chamber using the following calculation: total nuclei $=$ [sum from 16 squares/16 $\left.\left(6.25 \times 10^{4}\right)\right] \times($ total suspension volume $)$. In the 


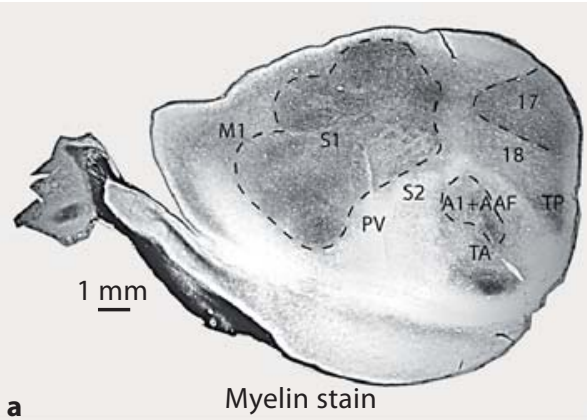

a

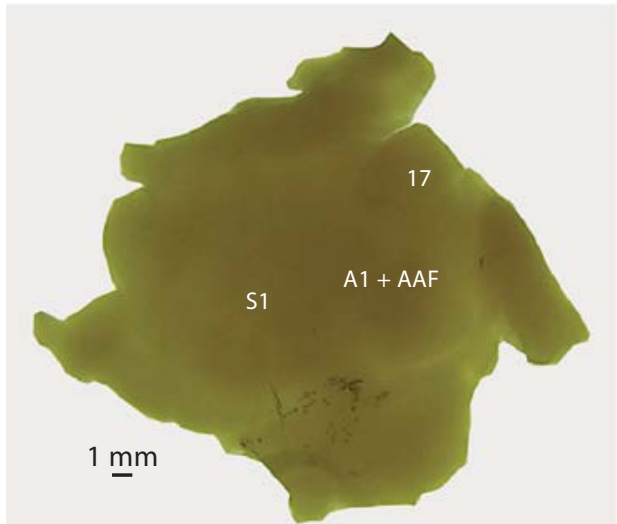

b Flattened hemisphere

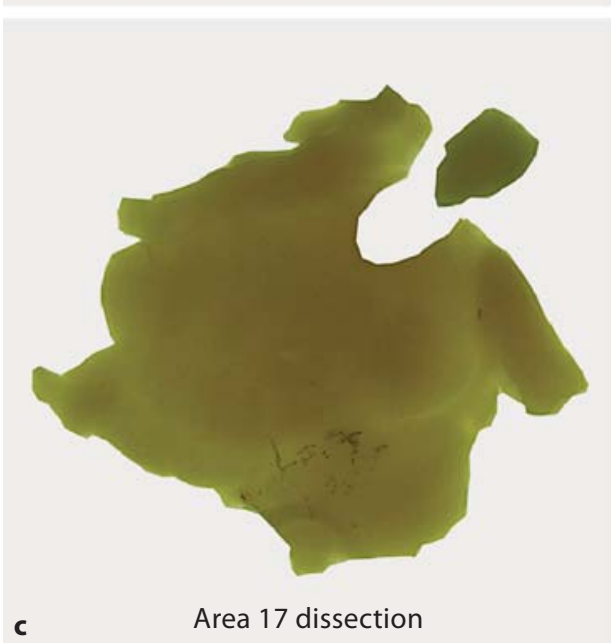

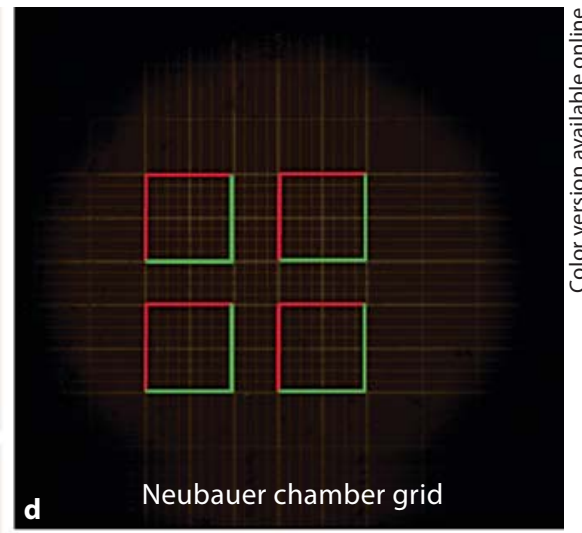
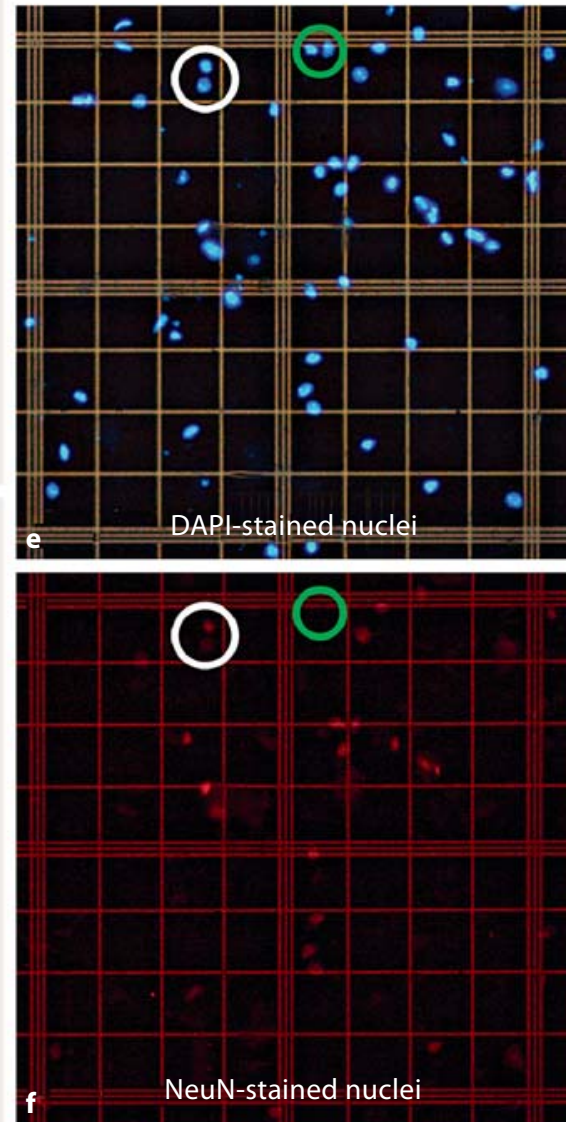

Fig. 2. Methods of the isotropic fractionator process. a Flattened cortical section stained for myelin to be used as reference for visualizing cortical area 17. Dotted lines denote primary areas. b Flattened cortical hemisphere with the medial wall reflected out is visualized on a light box. This back illumination allows the myelination pattern of the primary areas to be easily revealed. c Flattened cortical hemisphere on light box with area 17 dissected out. d Neubauer chamber grid used for counting cell nuclei. Boxes denote the area used for counting cell nuclei. A total of 16 boxes were counted for each specimen. Red lines denote the box edges excluded in counting and green lines denote box edges included in counting. e Digital images of Neubauer grid with DAPI-stained nuclei (blue) under UV illumination. White and green circles highlight two sets of nuclei. f Digital image of Neubauer grid with NeuN-stained nuclei under fluorescent red illumination. White circles highlight neuronal nuclei that can be visualized in both $\mathbf{e}$ and $\mathbf{f}$, while the green circle highlights the area in which nuclei were visualized only with DAPI under UV illumination. These nuclei would be classified as nonneuronal. Colors refer to the online version only. above calculation, $6.25 \times 10^{4}$ is the area of the squares multiplied by the volume of the squares. To estimate the percent of neurons in the sample, aliquots from the suspensions were immunocytochemically stained for anti-NeuN, and then at least 500 cells were evaluated for the presence or absence of the NeuN label. Groups of cells were first counted under UV illumination (all nuclei stained by DAPI; fig. 2e) and then the same groups were counted under red fluorescent illumination (neuronal nuclei stained by anti-NeuN; fig. 2f), yielding an estimate of the ratio of neuronal nuclei to total nuclei. From this estimate, the total number of neurons can be calculated using the following: total neurons = fraction of NeuN-positive nuclei $\times$ total DAPI-stained nuclei. The neuron density was calculated by the weight of the tissue sample as follows: neuron density = number of neurons/weight of structure.

Paired t tests were used to determine size and weight differences within a species between the left and right hemisphere pieces of area 17. No significant differences were found in area 17 between left and right hemispheres, prompting the combination of data from both hemispheres in further comparisons. F tests were used to examine differences in cortical field sizes between species. Significant F tests $(\alpha \leq 0.05)$ were followed up by Tukey's HSD test in order to assess which groups were significantly different (table 2). 
Table 2. F test, $\mathrm{p}$ values, group differences

\begin{tabular}{llcll}
\hline & F & & Significance & Group differences (Tukey's HSD) \\
\hline Total cells & $(2,25)$ & 12.141 & $<0.001$ & $\mathrm{~L}$ and $\mathrm{W}>\mathrm{G}$ \\
Total neurons & $(2,25)$ & 13.92 & $<0.001$ & $\mathrm{~L}$ and $\mathrm{W}>\mathrm{G}$ \\
Total nonneurons & $(2,25)$ & 10.366 & 0.001 & $\mathrm{~L}$ and $\mathrm{W}>\mathrm{G}$ \\
Nonneuron to neuron ratio & $(2,25)$ & 9.315 & 0.001 & $\mathrm{~L}>\mathrm{W}$ and G \\
Percent neurons & $(2,25)$ & 253.512 & $<0.001$ & $\mathrm{~L}<\mathrm{W}<\mathrm{G}$ \\
Neuron density & $(2,25)$ & 15.99 & $<0.001$ & $\mathrm{~L}<\mathrm{W}>\mathrm{G}$ \\
Nonneuron density & $(2,25)$ & 4.261 & 0.026 & $\mathrm{~L}$ and $\mathrm{W}>\mathrm{G}$ \\
Brain to body ratio & $(2,12)$ & 62.99 & $<0.001$ & $\mathrm{~L}<\mathrm{W}$ and G \\
\hline
\end{tabular}

$\mathrm{L}=$ Laboratory Norway rats; $\mathrm{W}=$ wild-caught Norway rats; $\mathrm{G}=$ Nile grass rats.

\section{Areal Border Tissue Processing, Reconstruction and \\ Data Analysis}

Animals were euthanized with a lethal dose of sodium pentobarbital $(250 \mathrm{mg} / \mathrm{kg})$ and perfused transcardially with $0.9 \%$ saline, followed by $3 \%$ paraformaldehyde ( $2 \%$ paraformaldehyde was used for the Nile grass rats) in 0.1 M phosphate buffer ( $\mathrm{pH}$ 7.4). After fixation, cortex was separated from subcortical structures, flattened and immersed in 30\% sucrose overnight and sectioned on a freezing microtome tangential to the cortical surface at a 40 $\mu \mathrm{m}$ thickness. This preparation allows the overall organization and positions of fields relative to each other to be determined. Although areal size data for the Norway rat groups are from a previous study [Campi and Krubitzer, 2010], the tissue was processed in the same manner as the Nile grass rat group.

Alternate series of cortical sections were reacted for myelin [Gallyas, 1979; Fang et al., 2005; Padberg et al., 2005; Campi et al., 2007] and for cytochrome oxidase (CO) [Carroll and Wong-Riley, 1984]. As these histological procedures have been described previously, we will only briefly describe them here. Cortical sections were split into two series: myelin and CO. The series reacted for myelin were placed in $5 \%$ formalin for 1 week and then transferred to distilled water overnight before processing using the method of Gallyas [1979]. Sections were transferred into a pyridine/acetic anhydride solution before the flattening procedure in distilled water. Next, sections were immersed in silver nitrate solution for $1 \mathrm{~h}$ before the developing step, which included several solutions (silver nitrate, ammonium nitrate, sodium carbonate and formalin). The final steps include washes in sodium thiosulfate and distilled water before mounting. The $\mathrm{CO}$ series was immediately processed after cutting. Sections were rinsed 3 times for $5 \mathrm{~min}$ in phosphate-buffered saline (PBS) and then transferred into a 3,3'-diaminobenzidine solution including cytochrome C, catalase, and PBS for 1-2 h. Sections were then rinsed 3 times in PBS before mounting.

Architectonic boundaries of the entire series of sections stained for myelin and $\mathrm{CO}$ were drawn using a camera lucida (Stemi SV6; Zeiss) or a projection microscope (Zeiss). The largest section from each hemisphere was selected for the outline for the final composite. Blood vessels and tissue artifacts were used to align individual sections during reconstruction. Boundaries for cortical areas were drawn by successive combination of cortical boundaries from individual sections throughout the entire series of sections into a final summary display. One section may not encompass the entirety of all cortical sensory areas due to minor differences across brains from the flattening and cutting process. Borders for the primary somatosensory area (S1), primary auditory area (A1), anterior auditory field (AAF), temporal anterior area and primary visual area (area 17) were drawn. These fields were chosen because they could be reliably and accurately identified in all of our animals (fig. 3).

Cortical area size measurements were derived using areal boundaries drawn in Adobe Illustrator from scanned summary displays. To normalize the data for comparison, the size of a particular cortical field was expressed as a percentage of the entire dorsolateral surface of the cortex. The dorsolateral surface of the cortex did not include the piriform cortex, olfactory bulbs or medial wall of the cortex.

Paired t tests were used to look for size differences between the left and right hemispheres. No significant difference between field sizes in left and right hemispheres was found so the data from both hemispheres were combined in further comparisons. Data sets for the Norway rat groups were taken from Campi and Krubitzer [2010]. F tests were used to examine differences in cortical field sizes between species. Significant F tests $(\alpha \leq 0.05)$ were followed up by Tukey's HSD test in order to assess which groups were significantly different (table 2). Regression analyses were done for the primary cortical areas as a function of neocortical area for the rat data from this experiment and for data from a previous experiment on California ground squirrels and Eastern gray squirrels [Campi and Krubitzer, 2010]. Minimal alterations were made in the brightness and contrast of all photomicrographs prepared in either Adobe Photoshop or Illustrator.

To determine whether differences in the size of cortical fields were simply due to allometry, as has been hypothesized in previous studies [e.g. Finlay and Darlington, 1995; Kaskan et al., 2005], we made calculations using the regression slopes provided by Kaskan et al. and our own data. Briefly, the regression slopes were used to calculate the predicted size and percentage of cortex devoted to a specific cortical area for each group based on the average neocortical sheet size for each group. Predictions for the size of each cortical area based on allometry were made using these calculations. 


\section{Results}

The overarching goal of these experiments was to compare the cellular composition of area 17/V1 in three rodent groups using a new technique, isotropic fractionation. However, we were also interested in obtaining data on overall brain size and cortical area size in the Nile grass rat, to compare to data previously collected for wild-caught and laboratory reared Norway rats [Campi and Krubitzer, 2010]. Below, we first briefly describe the architectonic appearance of primary sensory areas in all three species and the areal dimensions of the primary sensory areas. We then describe differences in body and brain weight, cortical field size and cellular composition of area 17 in all three groups of rats.

\section{Architectonic Appearance and Size of Primary}

\section{Sensory Cortical Areas}

Primary sensory areas contain a complete representation of the sensory receptor array for a specific modality (sense), have a highly myelinated layer IIIc, a densely cellpacked layer IV, and connections with modality (sensory)specific thalamic nuclei and other cortical areas. The degree of myelination was used to delineate cortical field boundaries (fig. 3). The relationship between architectonic boundaries and functionally defined cortical fields has been described previously for visual, somatosensory and auditory cortex in Long-Evans laboratory Norway rats [Adams and Forrester, 1968; Espinoza and Thomas, 1983; Malach, 1989; Roger and Arnault, 1989; Coogan and Burkhalter, 1993; Rumberger et al., 2001; Remple et al., 2003]. See table 3 for primary area means for each rat group. Further, in a recent study [Campi and Krubitzer, 2010], we have described in detail the architectonic appearance of sensory areas in both the laboratory Norway rat (Long-Evans) and the wild-caught Norway rat.

\section{Primary Visual Area - Area 17}

Brodmann's area 17 is coextensive with the functionally defined primary visual area (V1), is located caudomedially in the occipital pole and is easily delineated using a myelin stain as it is heavily myelinated and thus stains more darkly than surrounding areas (fig. 3). In all three rat groups, area 17 is a darkly myelinated wedgeshaped area that is homogeneous in appearance. Measurements of area 17 indicate that the mean proportion of dorsolateral cortex occupied by area 17 in the Nile grass rat is significantly larger $(8.15 \pm 0.23 \%$; mean \pm SEM) than both that of the laboratory Norway rat (7.91 $\pm 0.32 \%)$ and the wild-caught rat $(7.85 \pm 0.31 \%)$, which are not statistically significantly different from each other [Campi and Krubitzer, 2010]. The direction of difference here is in direct contrast to predictions of V1 size based on allometric scaling from the regression statistics in Kaskan et al. [2005], which would predict $9 \%$ of cortex devoted to V1 in the Norway rat groups and $8 \%$ in the Nile grass rat group (see below).

\section{Primary Somatosensory Area - S1}

The primary somatosensory area, $\mathrm{S} 1$ or area $3 \mathrm{~b}$, was similar in appearance in all animals examined. S1 is a large, irregularly shaped darkly myelinated area that is interspersed with lightly myelinated bands (fig. 3). In rats, this area contains the highly recognizable anatomically specialized 'barrel' cortex [Woolsey and Van der Loos, 1970; Welker, 1971; Woolsey et al., 1975]. S1 has a roughly similar shape in all three rodents that we examined: it is narrow at its medial pole and widens through the middle and lateral edge. The medial portion of S1 is roughly one half to one third of the rostral to caudal width of the lateral portion. Each species showed a different percentage of dorsolateral cortex occupied by S1, and these differences all reached statistical significance (table 2). Laboratory-reared Norway rats had the largest percentage $(28.05 \pm 0.53 \%)$, followed by the wild-caught Norway rat $(25.44 \pm 0.83 \%)$, and the Nile grass rat, with the smallest percentage of cortex devoted to S1 $(19.80 \pm 0.82 \%)$. The direction of difference here is in direct contrast to predictions of S1 size based on allometric scaling from the regression statistics in Kaskan et al. [2005], which would predict $11 \%$ of cortex devoted to S1 in the Norway rat groups and $13 \%$ in the Nile grass rat group (see below).

\section{Core Auditory Cortex}

The core auditory region in rodents generally contains two auditory fields with mirror representations of the species-specific auditory frequency range: the primary auditory area, A1 and the anterior auditory field, AAF. In the present study, we could readily identify a darkly myelinated oval-shaped core auditory area caudal to more lightly myelinated S2/PV in all species (fig. 3). A1 and AAF are not distinguishable based on myeloarchitecture and are considered here in aggregate as A1 + AAF. Measurements of A1 + AAF indicate that the mean proportion of dorsolateral cortex occupied by A1 + AAF in the laboratory Norway rat $(4.41 \pm 0.25 \%)$ is significantly smaller than that of the Nile grass rat $(5.78 \pm 0.47 \%)$. However, the relative areas of $\mathrm{A} 1+\mathrm{AAF}$ in both laboratory rat groups are statistically significantly larger than that of the wildcaught Norway rat $(3.24 \pm 0.26 \%)$. The direction of dif- 


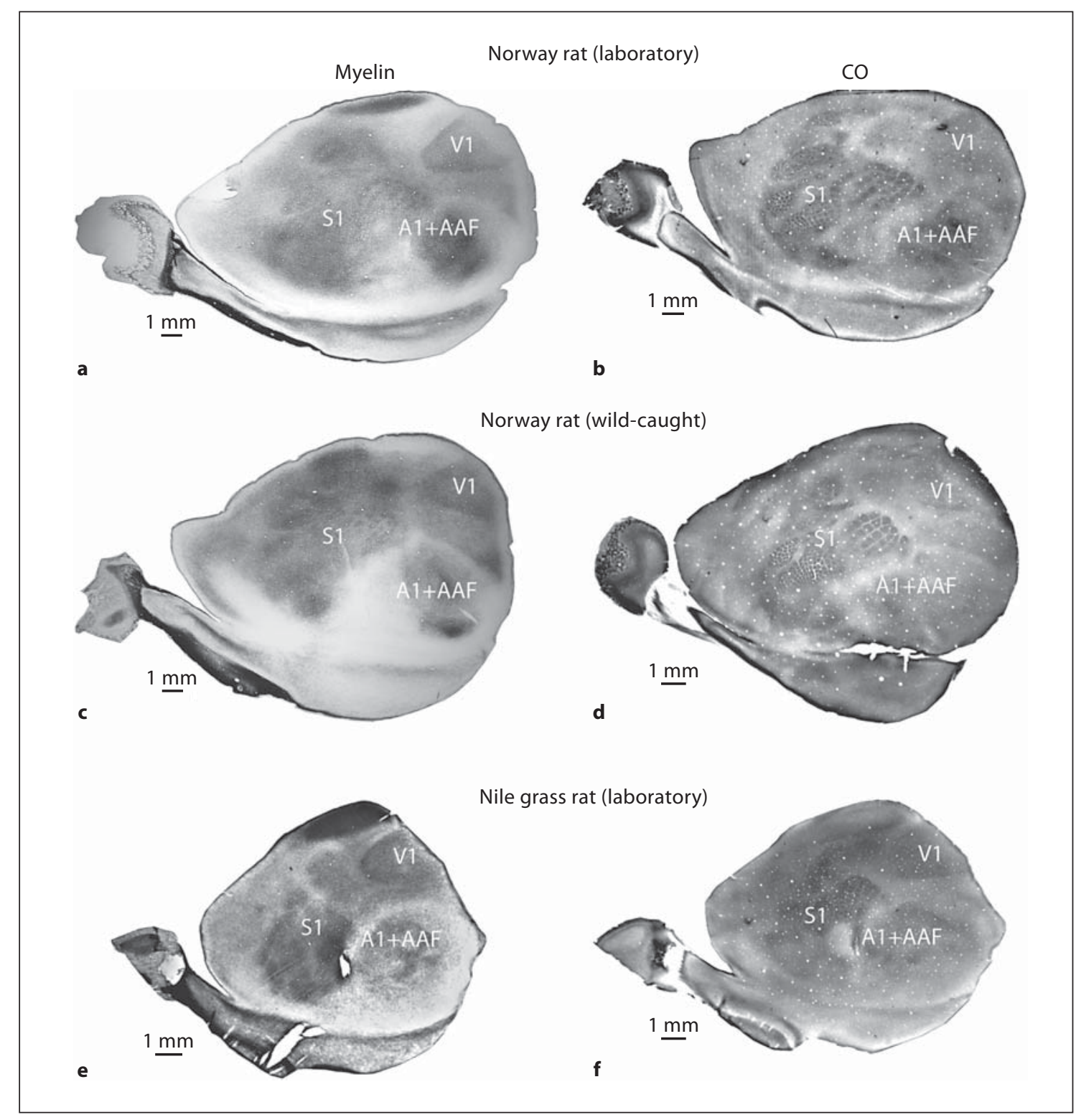

Fig. 3. Myeloarchitecture (left column) and CO reactivity (right column) in flattened cortical sections. Although the entire series of sections are examined, the digital images are of a single section for each stain from each rat group. $\mathbf{a}$ and $\mathbf{b}$ are from the laboratory Norway laboratory rat, $\mathbf{c}$ and $\mathbf{d}$ are from the wild-caught
Norway rat, and $\mathbf{e}$ and $\mathbf{f}$ are from the laboratory Nile grass rat. In all of these sections, area 17, A1 + AAF, and S1 can be readily identified. Data for the Norway groups come from Campi and Krubitzer [2010]. Rostral is to the left and medial is to the top. Scale bar $=1 \mathrm{~mm}$.
Table 3. Primary area measurement as mean percentage of dorsal cortex

\begin{tabular}{llll}
\hline & V1 & A1 + AAF & S1 \\
\hline Norway rat (laboratory) & $7.91 \pm 0.32$ & $4.41 \pm 0.25$ & $28.05 \pm 0.53$ \\
Norway rat (wild-caught) & $7.85 \pm 0.31$ & $3.24 \pm 0.26$ & $25.44 \pm 0.83$ \\
Nile grass rat (laboratory) & $8.15 \pm 0.23$ & $5.78 \pm 0.47$ & $19.80 \pm 0.82$ \\
\hline
\end{tabular}


ference here is in direct contrast to predictions of $\mathrm{A} 1+$ AAF size based on allometric scaling from the regression statistics in Kaskan et al. [2005], which would predict 2\% of cortex devoted to A1 + AAF in the Norway rat groups and $3 \%$ in the Nile grass rat group (see below).

Taken together, our data demonstrate that the mean percentage of cortex devoted to visual area 17 is significantly larger in the diurnal rat group than in nocturnal rat groups. On the other hand, the mean percentage of cortex devoted to somatosensory areas is significantly larger in the nocturnal rodents than in the diurnal rodent. Furthermore, the mean percentage of cortex devoted to the primary auditory cortex is significantly larger in the laboratory rat groups than in the wild-caught rat group.

\section{Body, Brain, Cortex and Area 17 Weights}

The Norway rat (laboratory and wild-caught) groups have a two-fold larger mean body weight, brain weight and area 17 weight compared to the Nile grass rat group means, and these differences are significant (see table 1 for means and table 2 for $F$ and $p$ values). Specifically, the laboratory Norway rat group body weight mean (508.3 \pm $150.2 \mathrm{~g}$ ) is significantly greater than the wild-caught Norway rat group mean $(170.1 \pm 16.6 \mathrm{~g})$, which is significantly greater than the laboratory Nile grass rat group mean $(104.3 \pm 9.3 \mathrm{~g})$ for body weight. The laboratory Norway rat group brain weight mean $(1.83 \pm 0.05 \mathrm{~g})$ is similar to the mean brain weight of the wild-caught Norway rat group (1.54 $\pm 0.05 \mathrm{~g})$, both of which are significantly greater than the mean brain weight of the Nile grass rat group $(0.87 \pm 0.02 \mathrm{~g})$. The laboratory Norway rat group cortex weight mean $(1.01 \pm 0.06 \mathrm{~g})$ is similar to the mean cortex weight of the wild-caught Norway rat group $(0.95 \pm 0.02 \mathrm{~g})$, both of which are significantly greater than the mean cortex weight of the Nile grass rat group $(0.50 \pm 0.01 \mathrm{~g})$. The laboratory Norway rat group area 17 weight mean $(0.022 \pm 0.002 \mathrm{~g})$ is significantly greater than the wild-caught Norway rat group mean $(0.018 \pm 0.001 \mathrm{~g})$, which is significantly greater than the laboratory Nile grass rat group mean $(0.010 \pm 0.0004 \mathrm{~g})$ for area 17 weight. Further, all three groups have a mean of $4 \%$ of the cortex (measured by weight) devoted to area 17. Thus, the weight of area 17 scales linearly with the weight of the cortex in all three groups. This is in accordance with a previous study comparing specific brain area weights in 6 rat strains that demonstrated a similar percentage (7-8\%) of cortical weight devoted to visual areas across all strains regardless of body and brain weight differences [Bennett et al., 1966].

\section{Cellular Composition of Area 17}

The Norway rat groups, which have larger brains and a larger area 17 by weight, also have significantly larger numbers of total cells, neurons, and nonneurons in area 17 compared to the Nile grass rats (see table 1 and fig. 4 for means and online suppl. table 3, for all supplementary material, see www.karger.com/doi/10.1159/000324862 for individual cases). The differences in total cells, neurons, and nonneurons in area 17 are about two-fold. Specifically, the laboratory Norway rat group mean total cells in millions $(2.01 \pm 0.18)$ is similar to the mean of the wild-caught Norway rat group (2.09 \pm 0.19$)$, both of which are significantly greater than the mean of the Nile grass rat group $(0.81 \pm 0.04)$. The mean total neurons in millions for the laboratory Norway rat group $(0.85 \pm$ $0.06)$ is similar to the mean of the wild-caught Norway group (1.02 \pm 0.09$)$, both of which are significantly greater than the mean of the Nile grass rat group $(0.42 \pm 0.03)$. However, the estimated nonneuron to neuron ratio is significantly larger in the laboratory Norway rat group (1.38 $\pm 0.08)$ compared to both the wild Norway rat group $(1.04 \pm 0.06)$ and the Nile grass rat group $(0.92 \pm 0.03)$.

The mean percentage of neurons among total cells in area 17 is significantly larger in the Nile grass rat (52 \pm $0.77 \%)$ compared to the wild Norway rat (49 $\pm 1.54 \%$ ), which is significantly larger compared to the laboratory Norway rat (43 $\pm 1.44 \%$; fig. 5). The wild-caught Norway rats have a significantly larger mean density of neurons (in thousands) per milligram of tissue (53 \pm 5 ) compared to the laboratory Norway rats (39 \pm 5 ) and the Nile grass rats $(41 \pm 3)$. The Nile grass rats have significantly smaller estimates of mean density in thousands of nonneurons per milligram of tissue $(38 \pm 2)$ compared to both laboratory Norway rats (53 \pm 5 ) and wild-caught Norway rats $(60 \pm 4)$.

Interestingly, when we looked at the brain to body weight percentage, the mean for the Nile grass rat group $(0.86 \pm 0.09 \%)$ was similar to the mean of the wildcaught rat group $(0.93 \pm 0.07 \%)$ and both were significantly larger compared to the mean of the laboratory Norway rat group $(0.34 \pm 0.04 \%$; fig. 5$)$. The wild-caught Norway rat group has a significantly larger mean encephalization quotient (EQ; $1.28 \pm 0.08)$ compared to the Nile grass rat group (1.05 \pm 0.09$)$, which is larger compared to the laboratory Norway rat group $(0.62 \pm 0.05)$.

Norway rat groups have significantly greater numbers of total cells, total neurons and total nonneurons in area 17 as well as a significantly larger area 17 by weight compared to the Nile grass rat group. The mean percentage of neurons in area 17 concurred with previous results [Her- 


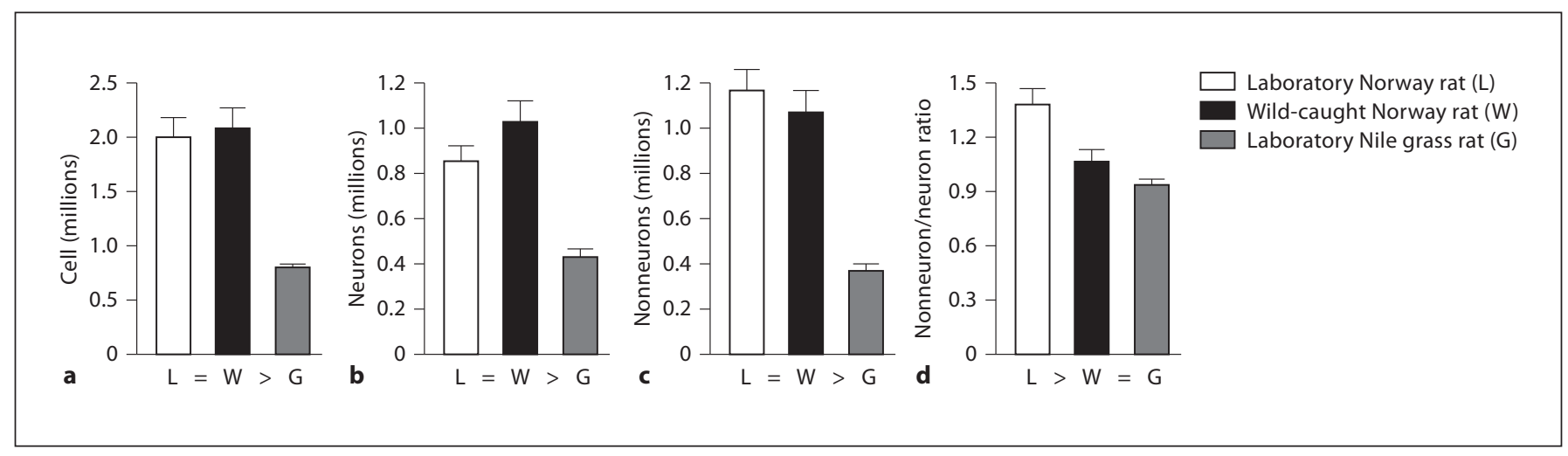

Fig. 4. Histograms showing estimated total cells (a), total neurons (b), total nonneurons (c) and the nonneuron/neuron ratio (d) in area 17 of each group. The $\mathrm{x}$-axis for all histograms shows the rat group, and the $y$-axis for histograms a-c shows the estimated numbers of cells in millions and for histogram $\mathbf{d}$ the ratio of nonneurons to neurons. Error bars represent the SEM. Significant differences between groups are indicated by the $>$ or $<$ symbol with abbreviations for each group beneath each histogram.
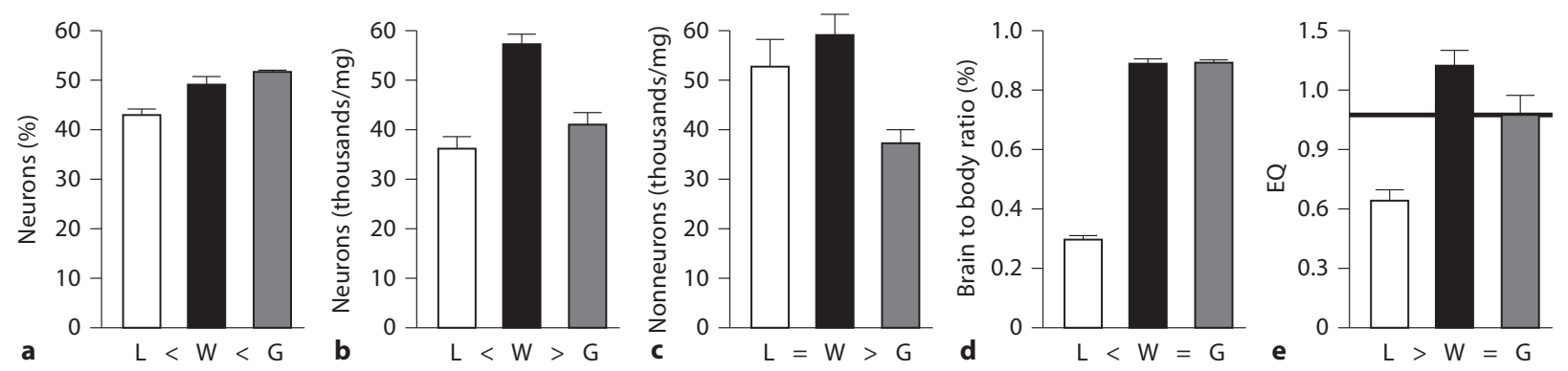

Fig. 5. Histograms showing the percent of neurons (a), the neuron density (b) and nonneuronal density (c) in area 17 of each group, brain to body weight \% (d) and EQ (e). The x-axis for all histograms shows the rat group, and the y-axis for histogram a is percent, for histograms $\mathbf{b}$ and $\mathbf{c}$ shows the estimated numbers of cells in millions, for histogram $\mathbf{d}$ percent and for histogram e ratio of expected brain weight to measured brain weight. It should be noted that the laboratory Norway rat group used for $\mathbf{d}$ and $\mathbf{e}$ had two very large rats $(>725 \mathrm{~g})$ which would account for the slightly greater difference here between the laboratory and wild-caught Norway rats compared to that in Campi and Krubitzer [2010]. The horizontal line represents a ratio of 1 . Conventions as in previous figure.
culano-Houzel et al., 2006] for brain size and percentage of neurons in rodent brains in that the Nile grass rat, with a smaller area 17 by weight, had the highest percentage of neurons followed by the wild-caught Norway rat and finally the laboratory Norway rat had the smallest percentage of neurons. The neuronal density in area 17 is as expected for the two laboratory rats, with the smaller brained Nile grass rat group having a higher neuronal density compared with the larger-brained laboratory Norway rat group. However, the significantly higher neuronal density in the wild-caught Norway rat group compared to both laboratory rat groups is in direct contrast to the allometric predictions. From these results, two patterns emerge with differences in primary sensory area size and cellular composition between wild-caught and laboratory rat groups and between diurnal and nocturnal rat groups. First, wild-caught rats have a significantly greater density of neurons in area 17 compared to the laboratory rats. The wild-caught rat group has a smaller percentage of dorsal cortex devoted to core auditory cortex compared to both of the laboratory rat groups. The wild-caught Norway group has a significantly greater EQ compared to the laboratory Norway rat group. Second, the diurnal rat group has a larger percentage of cortex devoted to V1 and 


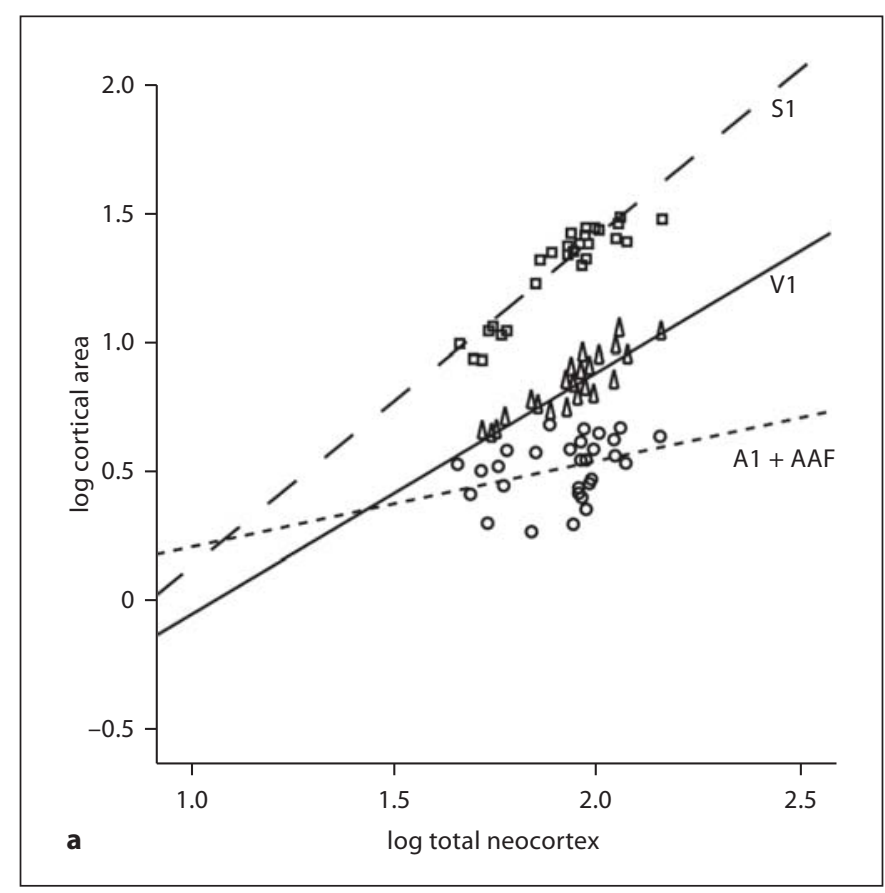

Fig. 6. Cortical area scaling in Norway and Nile rat groups (a) and California ground and Eastern gray squirrels (b) shown as log primary areas V1, S1, A1 + AAF (rats) and A1 + R (squirrels) regressed on log neocortex size. It should be noted that in the rat groups, S1 has the steepest slope compared with the squirrel groups in which V1 has the steepest slope. The equations and $\mathrm{r}^{2}$ values are as follows: $\mathbf{a} \mathrm{V} 1=0.969 \mathrm{x}-1.043, \mathrm{r}^{2}=0.827$; $\mathrm{S} 1=$

a smaller percentage of cortex devoted to S1 compared to the nocturnal rat groups. However, as specified above, the larger percentage of neurons in area 17 of the diurnal Nile grass rat group are due more to brain size differences rather than diel pattern. No other clear differences in cellular composition were observed between the nocturnal and diurnal rat groups. From these results, we conclude that the brains of the same species reared in radically different environments exhibit remarkable differences in cellular composition. However, small strain differences may also contribute to the cellular variation observed.

\section{Expectations due to Allometry}

In Kaskan et al. [2005], the linear relationship between cortical area size and neocortical size was examined in a meta-analysis using data from primates, monotremes, rodents, marsupials, insectivores, bats and tree shrews. A positive relationship between cortical area size and neocortical size was demonstrated such that as the neocortex enlarged so did primary cortical areas. In fact, V1 had the steepest slope (1.086) compared with the slope for A1

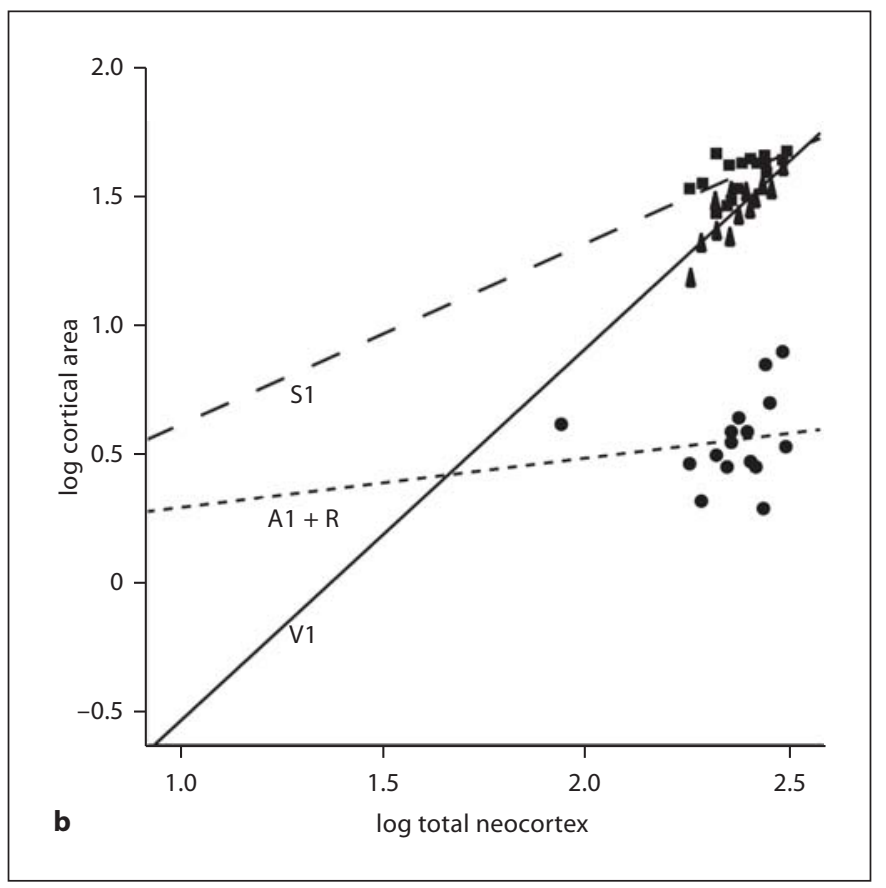

$1.274 \mathrm{x}-1.130, \mathrm{r}^{2}=0.0860 ; \mathrm{A} 1=0.332 \mathrm{x}-0.107, \mathrm{r}^{2}=0.096$. b V1 $=1.447 \mathrm{x}-2.003, \mathrm{r}^{2}=0.823 ; \mathrm{S} 1=0.698 \mathrm{x}-0.089, \mathrm{r}^{2}=0.453$; $\mathrm{A} 1=0.194 \mathrm{x}-0.085, \mathrm{r}^{2}=-0.036$. V1 individual points are depicted by triangles and the slope is a solid line, S1 individual points are depicted by squares and the slope is a long dashed line, and A1 individual points are depicted by circles and the slope is a short dashed line.
(0.697) and for S1 (0.664). Using the regression line from Kaskan et al., and the neocortex size from our data, V1 in the Norway rats should be $9 \%$, A1 should be $2 \%$, and S1 should be $11 \%$ of the neocortical sheet and V1 in the Nile grass rats should be $8 \%, \mathrm{~A} 1$ should be $3 \%$, and $\mathrm{S} 1$ should be $13 \%$ of the neocortical sheet. Our data do not match these predictions (see below). Before delving into statistical differences, we would like to point out an example in our raw data that illustrates the complicated relationship between cortical area size and neocortical size. In the Nile grass rat, the absolute size of the cortical sheet is about one half that of the wild-caught Norway rat $\left(\right.$ mean $=54.4 \mathrm{~mm}^{2}$ for Nile grass rat and mean $=95.4$ $\mathrm{mm}^{2}$ for wild-caught Norway rats); yet Nile grass rats have a larger A1 + AAF in absolute size as measured in square millimeters (mean $=3.12$ for Nile grass rat and mean $=3.04$ for wild-caught Norway rats). We calculated the regression line for our data (log transformed) and found that across rat groups, the steepest slope is observed for S1 (1.274) compared with the slope for A1 (0.332) and for V1 (0.969; fig. 6a). However, we also cal- 
culated the regression line for our data from wild-caught diurnal squirrels [Campi and Krubitzer, 2010] and found that in squirrels the steepest slope is observed for V1 (1.447) compared with the slope for A1 (0.332) and for S1 (0.698; fig. 6b). Thus, our regression analysis is similar to the results from our more straightforward percentage measurements in our different rat groups.

Neuronal density and percentages have been previously shown to scale with rodent brain size such that as brains get larger the density and percentage of neurons decrease [Herculano-Houzel et al., 2006]. However, the slopes are different in that the percentage of neurons decrease more steeply as brain size increases $(-0.223)$ and neuronal density decreases less steeply $(-0.475)$ in the cortex [Herculano-Houzel et al., 2006]. From these known values and brain size measurements, we would predict that the Nile grass rats will have a larger percentage of neurons and a greater density of neurons compared with the Norway rat groups. Our data on percentage of neurons fit this prediction, but the data on neuron density do not.

\section{Discussion}

Our results demonstrate that the differences in the size and cellular composition of cortical areas are beyond what would be predicted based on allometry alone and appear to have a consistent relationship with lifestyle and peripheral morphology. As in a recent study in our laboratory [Campi and Krubitzer, 2010], we observed that the proportion of cortex devoted to V1, by an areal measure of myeloarchitecture, is significantly greater in diurnal compared to nocturnal rodents. However, there were no clear cellular composition differences or differences in the percentage of cortical volume (by weight) in area 17 between nocturnal and diurnal rats. This is particularly interesting because of visual acuity differences between the diurnal and nocturnal rat groups discussed in the introduction. Nocturnal and diurnal rats have a different photoreceptor complement and recent studies in which changes in photoreceptor cells result in changes in behavioral discrimination abilities in adult color-blind monkeys demonstrate the ability of a sensory system to be coopted at the level of the receptor. This can happen first with relatively quick (within the animal's lifetime) downstream behavioral effects [Mancuso et al., 2009; Shapley, 2009]. It is possible then that in a lineage such as rats (Muridae) that have a nocturnal ancestor, the diurnal group has an anatomical change in the retina with commensu-

Comparative Studies of Area 17 in

Rodents rate physiological changes in the downstream structures such as the thalamus and cortex that do not necessarily translate into gross cellular composition changes. However, changes at other anatomical levels (molecular, spine density or connections) may be revealed with further examination. In fact, previous examinations of differences between nocturnal and diurnal mammals' visual systems demonstrate smaller receptive field sizes in V1 of diurnal mammals, which are presumably the result of anatomical differences at the cellular level [Hubel and Wiesel, 1968; Allman and Kaas, 1971; Hall et al., 1971; Espinoza and Thomas, 1983].

Because the Nile grass rats are laboratory reared, another possibility is that the full effects of retinal alterations may not be realized or may be negated as a result of the relatively impoverished environment. Recently, metrics similar to EQ have been developed to compare the number of neurons observed to the number of neurons expected in a given piece of tissue based on either the body or whole brain weight of the animal [HerculanoHouzel, 2007]. For example, the somatic neuronal quotient (SNQ) describes how the total number of neurons in the brain or a brain structure deviates from the expected for a rodent of a given body size and the encephalic neuronal quotient (ENQ) for a rodent brain of a given size. The wild Norway rat group has statistically greater ratios on all metrics comparing the density of neurons and allometric measures of neurons such as the SNQ and ENQ (see online suppl. fig. 1 and table 1 for equations) of V1 compared to both laboratory rat groups. Presumably, these differences in neuronal packing in combination with spine density differences demonstrated in environmental enrichment studies and cellular composition differences between laboratory and wild rodents would affect visual processing [Faherty et al., 2003; Kolb et al., 2003; Gelfo et al., 2009]. However, interpretation of the above ratios and EQ are difficult because these metrics do not take into consideration the absolute size of the animal brain and would therefore lead to similar cognitive abilities being predicted for small and large animals with similar EQs or SNQs. Herculano-Houzel [2007] suggests, using a measure of total neuronal excess (or the absolute number of neurons above that expected from body or brain mass), as a measure of cognitive abilities which they termed the neuronal index. Further, they predicted that higher neuronal indices (somatic neuronal index, SNI, and encephalic neuronal index, ENI; see online suppl. fig. 1 and table 2) would better correlate with cognitive abilities compared to EQ or SNQ. The present data could be interpreted to support this hypothesis - at least in V1 
for visual abilities. If we compare only the two laboratory rats (Norway and Nile grass rat groups) the diurnal Nile grass rat group has a significantly greater mean SNI and ENI for area 17 compared to the nocturnal Norway rat, which could partially (in combination with retinal composition differences) account for the greater visual acuity in this diurnal species compared to nocturnal Norway rats.

The neuronal density numbers we observed in our laboratory rats are in good agreement with previous studies on laboratory rats [Peters et al., 1985; Turner and Greenough, 1985]. Previous studies on neuronal density in rats in an enriched versus impoverished sensory environment have reported mixed results in the cortex. Some have reported higher neuronal densities of neurons in cortex in isolated versus enriched rats [Diamond et al., 1966; Diamond, 1967; Turner and Greenough, 1985] others have reported no significant difference in neuronal density in cortex between enriched and isolated rats [Diamond et al., 1975; for review, see Diamond, 2001]. This is in direct contrast to our results demonstrating higher neuronal density in area 17 of wild-caught rats compared to laboratory rats. However, several differences between our study and these previous studies may account for this difference. The greatest difference between our study and previous studies is in the use of wild-caught rats as the comparison (enriched) group. The environment of wild-caught rats is enriched for all the senses, and they likely have access to more and more varied visual stimuli than the classic 'enriched' laboratory rats. Sensory-deprivation studies have demonstrated that specific sensory deprivation from birth has an affect on the number of neurons in a specific sensory area. For example, rats reared in the dark showed greater apoptosis in areas $17,18,18 \alpha$ at postnatal day 21 [Papadopoulos and Michaloudi, 1999] and significantly lower neuronal density in the lateral geniculate nucleus compared with groups reared in standard light [Jameie et al., 2010]. One can reason from these studies that environmentally impoverished conditions from birth (i.e. standard laboratory environment) may result in greater apoptosis and therefore lower neuronal densities as adults compared with wild rats that developed in highly enriched natural conditions. Studies that reported lower densities of neurons in enriched versus impoverished environment rat groups started the manipulations after important critical periods (postnatal day 21) [for review, see Diamond, 2001]. It is likely that the underlying changes that occur in the brain with enrichment (addition or subtraction of neurons, glia and synapses or changes in soma/synapse size) changes throughout a lifetime and are dependent upon the time that the enrichment occurred. Taken to- gether, the data suggest that before naturally occurring apoptosis, cell numbers increase with enrichment while after apoptosis the size of cells and synapses are altered.

Presumably, these differences in neuronal packing in combination with connectivity differences demonstrated in environmental enrichment studies and cellular composition differences between laboratory and wild rodents would affect information processing. Thus, laboratory rodents must be considered highly altered neural models of their wild counterparts. Environmental conditions alter the molecular, cellular and functional operation of the network in sometimes unexpected ways. The alterations to the network generated through natural or enriched rearing conditions may result in emergent properties that would not exist in the simplified network of the laboratory animal.

Although the primary focus of our examination was on the visual cortex of rats, areal measurements in auditory and somatosensory cortex provide interesting data on the relationship between cortical organization and environment and sensory morphology. Both laboratoryreared rat groups (Norway and Nile grass rat) have significantly greater amounts of cortex devoted to A1 + AAF compared to the wild-caught Norway rat group. Further, the diurnal Nile grass rat group has a significantly smaller amount of cortex devoted to S1 compared to the nocturnal Norway rat groups. These findings are in good agreement with our previous studies in squirrels and wild-caught and laboratory rodents, but do not fit the expectations of cortical field size based on allometry. Furthermore, comparing the regression slopes from our data on both rat groups and squirrel groups (fig. 6) and regression slopes from Kaskan et al. [2005], from data across several mammalian orders, it would appear that cortical area scaling is dependent on the order of mammals examined and further dependent on the specific lineage. For example, the regression slopes of Kaskan et al. underestimate the percentage of cortex devoted to S1 in both the rat and squirrel groups by $8-10 \%$ and they underestimate the percentage of cortex devoted to V1 in the diurnal squirrel groups while they overestimate the percentage of cortex devoted to V1 in the nocturnal rat groups. Thus, other factors, including but not limited to sensory and motor specializations associated with lifestyle also appear to contribute to the amount of cortex devoted to a specific cortical area.

The slope differences between our data and those of Kaskan et al. [2005] may be explained by the combined data from several (but not all) mammalian orders in the former study, whereas our data are only from Rodentia. 
Further, the data in Kaskan et al. [2005] used to generate these predictions were gathered from studies using a variety of techniques including electrophysiology, cytoarchitectonics and neuroanatomy. Although these are all valid techniques, functional and cytoarchitectural boundaries do not always precisely coincide, and the rigor of data collection and analysis is different in different studies. Thus, the discrepancy between our data and previous data on allometric scaling of areas could be due to comparing cortical fields defined using different techniques, in different laboratories with different criteria for defining boundaries of cortical fields.

It should be noted that our data supporting a relationship between cortical field size, neuronal density and niche are supported by the specificity of cortical fields that are changing and the direction of that change. The highly visual, diurnal squirrel groups have a steeper slope for V1 scaling compared to the highly tactile, nocturnal rat groups, which have a steeper slope for S1 scaling (fig. 6). From these data we conclude that the size of cortical fields can be altered based on environmental demands. It is possible that the restrictions on movement and lack of visual diversity in a laboratory setting provide the impetus for greater reliance on audition in laboratory rodents. Thus, the distributions of cortical space allocation of sensory areas reflect, at least in part, the particular lifestyle and morphological specializations of each rodent group. The dissociation of cortical field size and neuronal composition in diurnal versus nocturnal rodents suggests that evolutionary changes in brains are nonlinear across levels of organization, and that the increase in cortical field size is due to factors other than the addition of more cells.

\section{Acknowledgements}

We thank Mark Blumberg for the Nile grass rat brain tissue. We thank Dylan Cooke and James Dooley for helpful comments on the manuscript.

This work was supported in part by the National Institutes of Health (R01-NS35103 to L.K.), the National Science Foundation (IOS - 0743924 to L.K.) the National Eye Institute (T 32 EY015387), a University of California, Davis Graduate Studies block grant to K.L.C. and a National Research Service Award (NS62633 to K.L.C.).

\section{References}

Adams AD, Forrester JM (1968): The projection of the rat's visual field on the cerebral cortex. Q J Exp Physiol Cogn Med Sci 53:327-336.

-Allman JM, Kaas JH (1971): Representation of the visual field in striate and adjoining cortex of the owl monkey (Aotus trivirgatus). Brain Res 35:89-106.

Arai JA, Feig LA (2010): Long-lasting and transgenerational effects of environmental enrichment on memory formation. Brain Res Bull, in press.

- Baroncelli L, Braschi C, Spolidoro M, Begenisic T, Sale A, Maffei L (2010): Nurturing brain plasticity: impact of environmental enrichment. Cell Death Diff 17:1092-1103.

Beaulieu C, Cynader M (1991): Effect of the richness of the environment on neurons in cat visual cortex. I. Receptive field properties. Brain Res Dev 53:71-81.

-Bennett EL, Diamond MC, Morimoto H, Hebert M (1966): Acetylcholinesterase activity and weight measures in fifteen brain areas from six lines of rats. J Neurochem 13:563-572.

Bland JM, Altman DG (1986): Statistical methods for assessing agreement between two methods of clinical measurement. Lancet i:307-310.

-Campi KL, Karlen SJ, Bales KL, Krubitzer L (2007): Organization of sensory neocortex in prairie voles (Microtus ochrogaster). J Comp Neurol 502:414-426.
Campi KL, Krubitzer L (2010): Comparative studies of diurnal and nocturnal rodents: differences in lifestyle result in alterations in cortical field size and number. J Comp Neurol 518:4491-4512.

-Cancedda L, Putignano E, Sale A, Viegi A, Berardi N, Maffei L (2004): Acceleration of visual system development by environmental enrichement. J Neurosci 24:4840-4848.

Carroll EW, Wong-Riley MT (1984): Quantitative light and electron microscopic analysis of cytochrome oxidase-rich zones in the striate cortex of the squirrel monkey. J Comp Neurol 222:1-17.

Chevret P, Denys C, Jaeger JJ, Michaux J, Catzeflis FM (1993): Molecular evidence that the spiny mouse (Acomys) is more closely related to gerbils (Gerbillinae) than to true mice (Murinae). Proc Natl Acad Sci USA 90:34333436.

Christensen J R, Larsen K B, Lisanby S H, Scalia J, Arango V, Dwork A J, Pakkenberg B (2007): Neocortical and hippocampal neuron and glial cell numbers in the rhesus monkey. Anat Rec 290;330-340.

-Collins CE, Young NA, Flaherty DK, Airey DC, Kaas JH (2010): A rapid and reliable method of counting neurons and other cells in brain tissue: a comparison of flow cytometry and manual counting methods. Front Neuroanat $4: 5$.
Coogan TA, Burkhalter A (1993): Hierarchical organization of areas in rat visual cortex. J Neurosci 13:3749-3772.

Coq J, Xerri C (1998): Environmental enrichment alters organizational features of the forepaw representation in the primary somatosensory cortex of adult rats. Exp Brain Res 121:191-204.

Davis DE (1947): The weight of wild brown rats at sexual maturity. Anat Rec 99:575.

Diamond MC (1967): Extensive cortical depth measurements and neuron size increases in the cortex of environmentally enriched rats. J Comp Neurol 131:357-364.

Diamond MC (2001): Response of the brain to enrichment. An Acad Bras Cienc 73:211-220.

Diamond MC, Law F, Rhodes H, Lindner B, Rosenzweig MR, Krech D, Bennett EL (1966): Increases in cortical depth and glial numbers in rats subjected to enriched environment. J Comp Neurol 125:117-125.

Diamond MC, Lindner B, Johnson R, Bennett EL, Rosenzweig MR (1975): Differences in occipital cortical synapses from environmentally enriched, impoverished, and standard colony rats. J Neurosci Res 1:109-119.

Douglas RM, Alam NM, Silver BD, McGill TJ, Tschetter WW, Prusky GT (2005): Independent visual threshold measurements in the two eyes of freely moving rats and mice using a virtual-reality optokinetic system. Vis Neurosci 22:677-684. 
Ebinger P (1974): A cytoarchitectonic volumetric comparison of brains in wild and domestic sheep. Z Anat Entwicklungsgesch 144:267302.

-Espinoza SG, Thomas HC (1983): Retinotopic organization of striate and extrastriate visual cortex in the hooded rat. Brain Res 272: 137-144.

Faherty CJ, Kerley D, Smeyne RJ (2003): A GolgiCox morphological analysis of neuronal changes induced by environmental enrichment. Brain Res Dev Brain Res 141:55-61.

Fang PC, Stepniewska I, Kaas JH (2005): Ipsilateral cortical connections of motor, premotor, frontal eye, and posterior parietal fields in a prosimian primate, Otolemur garnetti. J Comp Neurol 490:305-333.

-Finlay BL, Darlington FB (1995): Linked regularities in the development in evolution of mammalian brains. Science 268:1578-1584.

- Gaillard F, Bonfield S, Gilmour GS, Kuny S, Mema SC, Martin BT, Smale L, Crowder N, Stell WK, Sauve Y (2008): Retinal anatomy and visual performance in a diurnal conerich laboratory rodent, the Nile grass rat (Arvicanthis niloticus). J Comp Neurol 510:525538.

Gaillard F, Kuny S, Sauve Y (2009): Topographic arrangement of S-cone photoreceptors in the retina of the diurnal Nile grass rat (Arvicanthis niloticus). Invest Ophthalmol Vis Sci 50: 5426-5434.

Gallyas F (1979): Silver staining of myelin by means of physical development. Neurol Res 1:203-209.

-Gelfo F, De Bartolo P, Giovine A, Petrosini L, Leggio MG (2009): Layer and regional effects of environmental enrichment on the pyramidal neuron morphology of the rat. Neurobiol Learn Mem 91:353-365.

-Gilmour GS, Gaillard F, Watson J, Kuny S, Mema SC, Bonfield S, Stell WK, Sauve Y (2008): The electroretinogram (ERG) of a diurnal conerich laboratory rodent, the Nile grass rat (Arvicanthis niloticus). Vision Res 48:27232731.

- Hall WC, Kaas JH, Killackey H, Diamond IT (1971): Cortical visual areas in the grey squirrel (Sciurus carolinesis): a correlation between cortical evoked potential maps and architectonic subdivisions. J Neurophysiol 34:437-452.

-Herculano-Houzel S (2007): Encephalization, neuronal excess and neuronal index in rodents. Anat Rec (Hoboken) 290:1280-1287.

-Herculano-Houzel S, Lent R (2005): Isotropic fractionator: a simple, rapid method for the quantification of total cell and neuron numbers in the brain. J Neurosci 25:2518-2521.

-Herculano-Houzel S, Mota B, Lent R (2006): Cellular scaling rules for rodent brains. Proc Natl Acad Sci USA 103:12138-12143.

Hubel DH, Wiesel TN (1968): Receptive fields and functional architecture of monkey striate cortex. J Physiol 195:215-243.
Huchon D, Chevret P, Jordan U, Kilpatrick CW, Ranwez V, Jenkins PD, Brosius J, Schmitz J (2007): Multiple molecular evidences for a living mammalian fossil. Proc Natl Acad Sci USA 104:7495-7499.

Jameie SBE, Abdolrahmani M, Nobakht M (2010): Effects of total light deprivation on dorsal lateral geniculate nucleus of male neonate rats. Oman Med J 25:179-183.

Kaskan PM, Franco ECS, Yamada ES, de Lima Silveira LC, Darlington RB, Finlay BL (2005): Peripheral variability and central constancy in mammalian visual system evolution. Proc Natl Soc B 272:91-100.

Kolb B, Gibb R, Gorny G (2003): Experience-dependent changes in dendritic arbor and spine density in neocortex vary qualitatively with age and sex. Neurobiol Learn Mem 79: $1-10$.

Krubitzer LA, Kahn D (2003): Nature versus Nurture revisited: an old idea with a new twist. Prog Neurobiology 70:33-52.

Kruska D (1988): Effects of domestication on brain structure and behavior in mammals. Hum Evol 3:473-485.

Lovtrup-Rein H, McEwen BS (1966): Isolation and fractionation of rat brain nuclei. J Cell Biol 30:405-415.

Malach R (1989): Patterns of connections in rat visual cortex. J Neurosci 9:3741-3752.

Mancuso K, Hauswirth WW, Li Q, Connor TB, Kuchenbecker JA, Mauck MC, Neitz J, Neitz M (2009): Gene therapy for red-green colour blindness in adult primates. Nature 461:784787.

-Mahmoud AM, Depoorter B, Piens N, Comhaire FH (1997): The performance of 10 different methods for the estimation of sperm concentration. Fertil Steril 68:340-345.

- Mohammed AH, Zhu SW, Darmopil S, HjerlingLeffler J, Ernfors P, Winblad B, Diamond MC, Eriksson PS, Bogdanovic N (2002): Environmental enrichment and the brain. Prog Brain Res 138:109-133.

Mouton, P R (2002): Principles and Practices of Unbiased Stereology: an Introduction For Bioscientists. Baltimore, Johns Hopkins University Press.

Padberg J, Disbrow E, Krubitzer L (2005): The organization and connections of anterior and posterior parietal cortex in titi monkeys: do New World monkeys have an area 2? Cereb Cortex 15:1938-1963.

Papadopoulus GC, Michaloudi HC (1999): Apoptosis in the visual system of normal and dark-reared rats. Brain Res 838:214-217.

Perry JS (1946): The reproduction of the wild brown rat (Rattus norvegicus Erxleben). Proc Zool Soc Lond 115:19-46.
Peters A, Kara DA, Harriman KM (1985): The neuronal composition of area 17 of rat visual cortex. III. Numerical considerations. J Comp Neurol 238:263-274.

Prusky GT, Reidel C, Douglas RM (2000): Environmental enrichment from birth enhances visual acuity but not spatial learning in mice. Behav Brain Res 114:11-15.

- Remple MS, Henry EC, Catania KC (2003): Organization of somatosensory cortex in the laboratory rat (Rattus norvegicus): evidence for two lateral areas joined at the representation of the teeth. J Comp Neurol 467:105118.

Roger M, Arnault P (1989): Anatomical study of the connections of the primary auditory area in the rat. J Comp Neurol 287:339-356.

Roll U, Dayan T, Kronfeld-Schor N (2006): On the role of phylogeny in determining activity patterns of rodents. Evol Ecol 20:479-490.

Rosenzweig MR (1966): Environmental complexity, cerebral change, and behavior. Am Psychol 21:321-332.

Rumberger A, Tyler CJ, Lund JS (2001): Intraand inter-areal connections between the primary visual cortex V1 and the area immediately surrounding V1 in the rat. Neuroscience 102:35-52.

Shapley R (2009): Vision: Gene therapy in colour. Nature 461:737-739.

Steppan SJ, Storz BL, Hoffmann RS (2004): Nuclear DNA phylogeny of the squirrels (Mammalia : Rodentia) and the evolution of arboreality from c-myc and RAG1. Mol Phylogenet Evol 30:703-719.

-Sukcharoen N, Ngeamjirawat J, Chanprasit Y, Aribarg A (1994): A comparison of Makler counting chamber and improved Neubauer hemocytometer in sperm concentration measurement. J Med Assoc Thai 77:471-476.

Turner AM, Greenough WT (1985): Differential rearing effects on rat visual cortex synapses. I. Synaptic and neuronal density and synapses per neuron. Brain Res 329:195-203.

Welker C (1971): Microelectrode delineation of fine grain somatotopic organization of $(\mathrm{SmI})$ cerebral neocortex in albino rat. Brain Res 26:259-275.

World Health Organization (1999): WHO Laboratory Manual for the Examination of $\mathrm{Hu}$ man Semen and Sperm-Cervical Mucus Interactions, ed 3. Cambridge, Cambridge University Press.

Woolsey TA, Van der Loos H (1970): The structural organization of layer IV in the somatosensory region (SI) of mouse cerebral cortex. The description of a cortical field composed of discrete cytoarchitectonic units. Brain Res 17:205-242.

-Woolsey TA, Welker C, Schwartz RH (1975): Comparative anatomical studies of the SmL face cortex with special reference to the occurrence of 'barrels' in layer IV. J Comp Neurol 164:79-94. 